\title{
Management of intra-vitreal cysticercosis
}

\author{
Yadav SK, Winter I, Singh SK \\ Biratnagar Eye Hospital, Biratnagar, Morang, Nepal
}

\begin{abstract}
Background: South East Asia is an endemic zone for cysticercosis. Oocular cysticercosis is emerging as a common problem which if untreated can lead to severe visual loss in 3-5 years.

Cases: We describe here a case series of three patients with intravitreal cysticercosis and managed by surgical cyst removal by pars plana vitrectomy.

Observation: The procedure was successful in all cases to maintain anatomical integrity of the globe with some functional gain of vision.

Conclusion: Surgical intervention in ocular intravitreal cysticercosis is an acceptable approach with good results.
\end{abstract}

Key words: cysticercosis, ocular cysticercosis, intravitreal cysticercosis

\section{Introduction}

Ocular cysticercosis in South East Asia is an increasingly common problem caused by infestation of larval form of pork tapeworm Cysticercus cellusae (Duke-Elders S \& Perkins ES, 1966). It is different from teaniasis which is caused by adult tape worm Taenia solium. History goes back to 1829 when Scholl and Soemmerring first discovered a live Cysticercus cyst in an anterior chamber, since then several authors described and reported isolated cases (Madan et al, 1991; Grover \& Puri,1996; George at al, 1999) from different parts of the world. But clinical studies (Shekhar \& Lemke 1997; Puri \& Grover, 1998; Shekhar \& Honavar 1999; Shrestha JB et al, 2008; Basu S et al, 2009) of various presentations, diagnostic modalities and treatment options are limited. Cysticercosis has a worldwide distribution but is endemic in South East Asia, Latin America, Africa and Eastern Europe (Schantz PM). Against common beliefs it can affect both vegetarians \& non -vegetarians (Kean BH, Sun $\mathrm{T}$, Ellsworth RB) and is seen in people belonging to low socio- economic groups associated with poor hygiene(Atul K, Kumar TH).

Received: 02. 06.2009 Accepted: 28.06.2009

Correspondence and reprint request to: Dr Sanjeev Kumar Yadav, MD

Vitreo - Retina Services

Biratnagar Eye Hospital, Morang

Nepal

Email: drsanjeevyadav@gmail.com
We are presenting here a series of three cases of intravitreal cysticercosis seen over a 2 year period (2007-2009) in Biratnagar Eye Hospital. All the cases were managed surgically. After freeing the cyst from vitreous attachment by three ports 20 gauge pars plana vitrectomy (PPV) it was removed by suction with the vitreous cutter. Oral albendazole 400mg BD for 1 month. \& oral prednisolone in tapering dose starting with $1 \mathrm{mg} /$ $\mathrm{kg}$ body weight per day were given to each patient. Blood counts, Stool examination and head CT scan had been performed in all cases but Serological tests were not carried out due to lack of test availability and limited diagnostic value. Symptomatic general body review including CNS was within normal limit in all cases.

\section{Case 1}

A 22 yr old male from Assam / India presented in our hospital with painless progressive loss of vision since 2 months in his left eye. Visual acuity was $6 / 6$ and $6 / 24$ (right and left eye respectively). Slit lamp examination revealed no abnormality. Ophthalmoscopy of his left eye showed intravitreal cysticercosis temporal to fovea, which was confirmed by B-scan ultrasonography. History of pork diet was absent. Complete blood counts, stool test for microscopy/parasites and CT- brain revealed no abnormality.

Oral albendazole 400mg B.D. with oral prednisolone was started and PPV carried out after 3 days. The 
cyst was removed with vitrectomy probe after clearing all the adherent vitreous. Endolaser was applied to the ischemic parts of the retina. Silicon oil was used as a vitreous substitute and also to keep the edematous retina in the macular region reattached.

Post operative and follow up period was unremarkable. Repeated blood tests showed normal findings. Repeated stool tests were negative for any type of ova or cyst. Silicone oil removal was done after 6 months without any complications. The final visual acuity stabilized at $6 / 12$ in the operated eye.

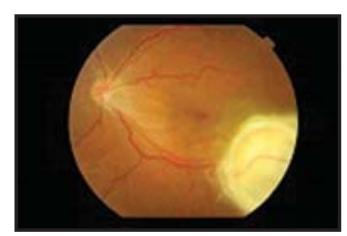

Fig. 1 case 1: Fundus photograph of left eye showing cyst temporal to fovea

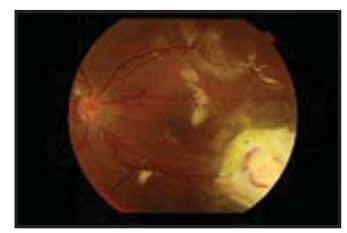

Fig. 3 case 1: Post operative photograph after cyst removal. Retina well attached under silicon oil

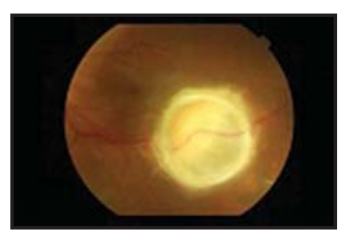

Fig. 2 case 1: Central view of cyst with inflammatory reaction

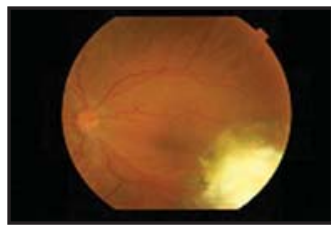

Fig. 4 case 1: Scarred lesion in the previous cyst area after silicone oil removal. Retina is well attached.

\section{Case 2}

A 35-year old female from Jhapa / Nepal presented with HM visual acuity in right eye and 6/6 in left eye. Her complaints were progressive loss of vision in right eye from last few months with pain during last few days prior to her visit. On examination intravitreal cyst was found in her right eye with accompanying vitritis. B-scan confirmed the diagnosis of cysticercosis. History of pork meal was present. Blood and stool tests as well as brain CT-scan found no abnormalities. Oral Steroids and albendazole were started and PPV done with cyst removal by vitrectomy probe as in case 1 after vitritis had resolved. Post operative and follow up period in this case too were uneventful with normal blood counts and stool tests. She is in our regular followup and her best corrected vision is $6 / 36$. Silicon oil has not yet removed.

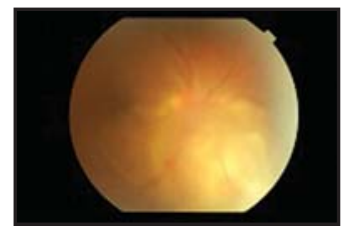

Fig. 5 case 2: Fundus photograph of right nasally with hazy media due to vitritis

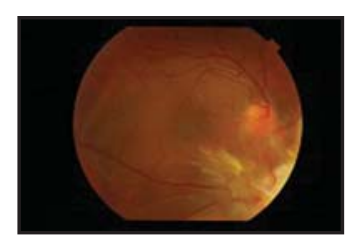

Fig.7 Case 2: Post operative photograph of attached retina under silicon oil. eye showing cyst

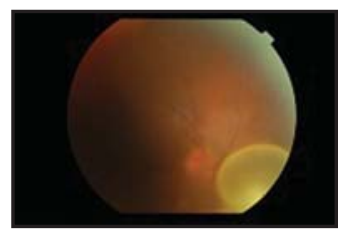

Fig.6 Case 2: After steroid/albendazole therapy and partial resorption of vitritis. Cyst nasally is clearly visible.

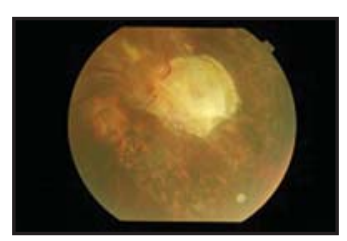

Fig. 8 Case 2: Original area of cyst lodgment now with scarring and laser marks.

\section{Case 3}

A 25 year-old male patient from Damak / Nepal presented with progressive painless loss of vision in left eye since last one month with presenting visual acuity of 6/6 and counting fingers (CF) in one meter in the right and left eyes respectively. During examination, we diagnosed intravitreal cysticercosis with associated central tractional retinal detachment. History of pork diet was present. CT brain identified multiple cysts in the brain parenchyma but surprisingly without any symptoms. We managed the case surgically similarly like first two patients. Due to brain parenchyma involvement the patient was initially admitted to a neurosurgical ward for observation during the first two days of treatment with oral albendazole and steroids. Post operative period in this case was also uneventful. On first postoperative day his vision was $6 / 60$.

A long term follow up is still pending. 


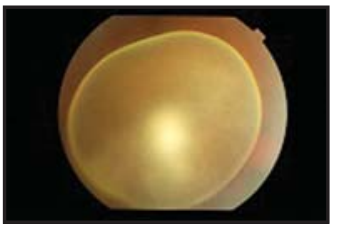

Fig.10 Case 3:

Fig. 9 Case 3:

Tractional retinal detachment

associated with cysticercosis

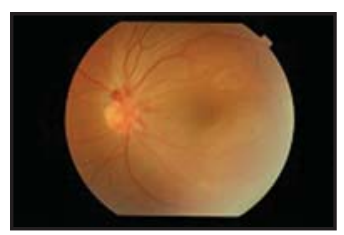

Fig.11 Case 3: Post operative photograph showing retina attached under silicone oil. Intravitreal cyst with scolex inside.

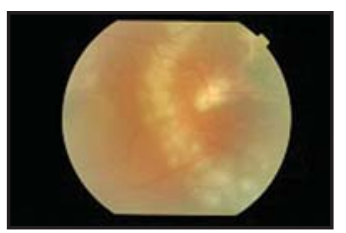

Fig.12 Case 3: Post operative period showing area of original cyst and relieved traction with scarring and laser marks.

\section{Discussion}

The ocular cysticercosis is an important cause of visual morbidity in this region. More work is needed to provide ophthalmologists with proper management guidelines. Our experience is small but nevertheless demonstrated typical clinical pictures of the disease. All our cases presented with a unilateral ocular disease and were consistent with other studies (Grover \& Puri, 1996; George et al, 1999)' carried out in different parts of the word. For management strategies conflicting reports exist in the literature. Some reports from India advocate early surgical intervention (Shekhar \& Honavar, 1999; Sekhar \& Lemke, 1997) and our experience is also the same. In all our cases, we surgically removed the cyst by PPV under the coverage of steroids and albendazole. This technique was found simple and reliable. It stabilizes the anatomy of the affected eye and prevents the patient from experiencing severe visual loss or even blindness which would otherwise occur if left untreated. The CNS involvement which can cause severe implication is considered as a rare association. But our case series showed involvement in one patient. We, therefore, advocate CT scan of the head mandatory in all cyst cases to prevent any devastating complication in time.

\section{References}

Basu S, Muthusami S, Kumar A (2009). Ocular cysticercosis: an unusual cause of proptosis. Singapore Med J; 50:e309-11.

Duke-Elders S, Perkins ES (1966). Inflammations of the uveal tract: uveitis. In: System of Ophthalmology. St. Louis: Mosby, chap 3, Vol 9: 478-87.

Schantz PM (2002). Taenia solium cysticercosis: an overview of global distribution and transmission. In: From Basic to Clinical Science. CABI Publishing: 63-74.

Kean BH, Sun T, Ellsworth RB (1991). Cysticercosis. In: Colour atlas/ text of ophthalmic parasitology. Editors: Kean BH, Sun T. Ellsworth RBIgaku-Shoin, Medical Publishers Inc: New York: 173-81.

Atul K, Kumar TH, Mallika G, Sandip M (1995). Sociodemographic trends in ocular cysticercosis. Acta Ophthalmol Scand; 73:438-41.

Puri P \& Grover AK (1998). Medical management of orbital myocysticercosis: a pilot study. Eye;12: 795799.

Shekhar GC \& Honavar SG ( 1999). Myocysticercosis: Experience with imaging and therepy. Ophthalmology; 106: 2336-2340.

Sekhar GC \& Lemke BN (1997): Orbital cysticercosis. Ophthalmology; 104: 1559-1604.

Grover AK \& Puri P (1996). Orbital myocysticercosis presenting as subconjunctival abscess. Ind $\mathrm{J}$ Ophthalmol; 44: 229-231.

George AE, Biswas J, Agarwal R, Kumarasamy N, Solomon S (1999): Subretinal cysticercosis in a patient with AIDS: treatment with xenon arc photocoagulation. Retina; 19: 467-468.

MadanVS, Dhamija RM, Gill HS, Boparai MS, Souza PD, Sanchete PC, Bhardwaj JR (1991): Optic nerve cysticercosis: a case report. J Neurol Neurosurg Psychiatry; 54: 470- 471.

Source of support: nil. Conflict of interest: none 